\title{
The historical change of brainstem glioma diagnosis and treatment: from imaging to molecular pathology and then molecular imaging
}

\author{
Liwei Zhang ${ }^{1,2,3^{*}}$, Chang-cun Pan ${ }^{1,2,3}$ and Deling Li $i^{1,2}$
}

\begin{abstract}
Understanding a process from shallow to deep is necessary for controlling and even curing diseases. The history of diagnosis and treatment of brainstem gliomas vividly reflects this process. The development of neuroimaging plays a great role in tumor treatment at different periods, including the period when brainstem gliomas were regarded as an homogenous incurable disease, and currently it is considered as an entity with high heterogeneity. Presently, it is not enough to just rely on the conventional neuroimaging techniques to determine the anatomic location of a tumor and its relationship with normal tissues. The development of molecular genetics and molecular imaging further promotes the progress of individualized and precision diagnosis and treatment in brainstem gliomas. In this paper, we summarize the evolution of brainstem glioma radiological classification mainly focusing on the aspects of imaging and surgical treatment. In the meanwhile, we reviewed the recent progresses in the fields of molecular genetics and molecular imaging.
\end{abstract}

Keywords: Brainstem gliomas, Microsurgery, Radiological classification, Molecular genetics, Molecular imaging

\section{Introduction}

The breakthrough of the "no man's land"

In the 1960s, the brainstem was still the forbidden region for surgery, and the mortality rate of operation on brainstem tumors was nearly $100 \%$. In 1969, Maston [1] stated that "regardless of specific histology, brainstem gliomas must be classified as malignant tumors since their location itself renders them inoperable."

Hoffman et al. [2] reported a group of benign brainstem gliomas with distinct clinical and pathological manifestations, which originate at the fourth ventricle bottom of the dorsal part of brainstem, and grow into the fourth ventricle through the ependyma. Therefore, this kind of tumor was named as the dorsal exophytic brainstem glioma. Due to the early age of onset, the common clinical symptoms of this tumor include elevated intracranial pressure (ICP)

\footnotetext{
*Correspondence: zhangliweittyy@163.com

'Department of Neurosurgery, Beijing Tiantan Hospital, Capital Medical University, Tiantanxili 6, Dongcheng District, Beijing 100050, China

${ }^{2}$ China National Clinical Research Center for Neurological Disease, Tiantanxill 6, Dongcheng District, Beijing 100050, China

Full list of author information is available at the end of the article
}

caused by hydrocephalus and failure to thrive. After total resection, patients can achieve a relatively long survival without routine postoperative radiotherapy, and most residual tumors are stable. The further operation can be considered when the tumor is relapsed. Although, pathological studies showed that most patients reported by Hoffman et al. belong to WHO I-II type fibrillary astrocytoma (only two cases of patients are ganglioglioma) [2], the most common pathological type for the dorsal exophytic brainstem gliomas and diffuse intrinsic pontine gliomas (DIPGs) is pilocytic astrocytoma and fibrillary astrocytoma, respectively [3]. These findings reported by Hoffman et al. have the epoch-making significance and subvert the traditional concept that brainstem is the forbidden region for surgery for many years.

\section{Review}

From "imagination" to "visualization"

There are four main tasks including localization diagnosis, qualitative diagnosis, guiding treatment, and dynamically monitoring of disease evolution following the treatment, during the development of neuroimaging 
technology. Regarding the treatment of brainstem gliomas, each technology, such as pneumoencephalography, iodipin ventriculography, cerebral angiography, CT and MRI, has made historic contributions for the achieving of these four tasks. Following the period of "imagination", it is currently in the "visualization" period for the diagnosis and treatment of brainstem gliomas.

The "imagination" refers to a kind of preliminary diagnosis made by the doctors based on the patient inquiry and neurological examination before the presence of neuroimaging techniques. Doctors need to comprehensively use their knowledge of physiology and anatomy to reconstruct and understand the precise location and three-dimensional shape of tumor and its relationship with surrounding normal tissues. Therefore, it was a great progress from 1917, when a variety of contrast enhancement techniques have been developed successively. These imaging techniques can detect the indirect signs of space occupying lesion in brainstem, improve the accuracy of diagnosis and provide a relatively objective basis for surgical exploration together with neurological examinations. However, these imaging techniques are invasive methods, which are not able to provide the patho-histological diagnosis, guide the treatment, and monitor the effects of treatment, except for providing the indirect signs of space occupying lesion. After the clinical use of CT for diagnosis since 1971, the diagnosis levels were further promoted. CT is a tomography that can indicate the lesion density and directly show brainstem tumors. In 1987, Stroink et al. [4] classified the brainstem tumor into four types based on CT findings of the lesions and the intraoperative findings, including (I) dorsal exophytic gliomas (iso-density, obvious enhancement), (II) diffuse intrinsic brainstem tumors (can be divided into IIa, low density and no enhancement, and IIb, high density and enhancement, with exophytic component, (III) focal intrinsic cystic tumor (cyst wall enhancement), and (IV) focal intrinsic solid tumors (iso-density, markable enhancement). Although this classification can not reflect the whole imaging manifestations of the brainstem gliomas, it can predict the expected surgical effect and prognosis to a certain extent. Moreover, it has the basic parameters (growth pattern and tumor-imaging features) for imaging classification in brainstem gliomas. The growth pattern can be divided into exogenous and endogenous types, and the latter one can be further divided into diffuse and focal types. Tumor imaging features on $\mathrm{CT}$ can be showed by the changes of density, with or without enhancement and cystic degeneration.

Conceptually, this classification reflects a profound understanding on brainstem gliomas by neurosurgeons in the 1980s. However, due to the fact of low resolution of soft tissues, being unable to do three-dimensional reconstruction, low ability to reflect tumor information in addition to density and enhancement, as well as bone artifacts of posterior fossa, CT as an imaging tool did not make this idea bright. Rapidly, MRI replaced CT and became the main tool for diagnosing and treating brainstem gliomas due to its several advantages, such as high resolution in soft tissues, three-dimensional reconstruction at any angle for precise localization, no radioactivity, imaging sequence diversification, and no bone artifacts.

According to the MRI findings and the observations during the operation of brainstem gliomas, Epstein proposed a classification framework in 1985, where brainstem glioma was classified into exophytic, intrinsic, and disseminated types [5]. The exophytic type has three subtypes, including diffuse, focal, and cervicomedullary subtypes, and the intrinsic type can be divided into subtypes of cerebellopontine angle (CPA), brachium points, and fourth ventricle. Epstein [6] reported the surgical treatment experience of 34 cases of intrinsic brainstem gliomas in 1986. In this report, all the diffuse tumors were high-grade gliomas (WHO III-IV), all the cervicomedullary tumors were low-grade gliomas (WHO I-II), and the major focal tumors were low-grade tumors. There were no surgical benefits in patients with high-grade tumors, while neurological dysfunction was significantly improved in patients with cervicomedullary tumors after the operation, and their diseases were stable for 2 to 5 years postoperation. Therefore, it is positively suggested to have the surgical treatment for the patients with cervicomedullary tumors, but not for the patients with focal tumors which are highly suspected to be high grade $[6,7]$.

The classification of Epstein focuses on the growth pattern based on the hypothesis that different pathological types of tumors have different growth patterns. Different growth patterns determine whether the patients should receive the surgical treatment. Therefore, the cervicomedullary type of brainstem gliomas in this classification does not only represent the location but also and more importantly refer a specific growth pattern. This kind of tumor is benign, which grows up from the upper cervical spinal cord, but its growth is limited by the pyramidal decussation, decussation of lemniscus and pia mater, which change the growth direction and make the tumor grow into the fourth ventricle through the ependyma. For these reasons, the prognosis of this tumor is similar with the intramedullary low-grade tumors, and surgical treatment has good outcomes [8]. In the classification of Epstein, a dorsal exophytic tumor is one kind of focal benign tumors growing in the medulla oblongata, and its growth is also limited by the surrounding fibers, thus changing the growth direction into the fourth ventricle through the ependyma [8]. The prognosis of this tumor is good, and the operation can even cure the disease. The proposal that the growth of low-grade tumors is limited by the surrounding normal tissues is supported and confirmed by Scherer [9]. 
The safety and efficacy of surgical treatment for dorsal exophytic and cervicomedullary type of brainstem gliomas has been recognized in the late 1980s promoted by Hoffman and Epstein [2, 6, 7]. Both types of tumors escape from the concept that operation is not suitable for brainstem gliomas. DIPG is one kind of brainstem gliomas with the highest incidence, shortest survival time, and highest difficulty for therapy [10]. Due to the fact that operation does not exhibit any effect on this tumor, subsequent studies focused on radiotherapy and chemotherapy. Since the early 1990s, stereotactic biopsy, as an invasive tool to acquire histopathological diagnosis, has been replaced by the noninvasive MRI examination, because tissue obtained through biopsy cannot represent the whole of the tumor, thus the diagnosis may be misleading; besides, knowing the histopathological diagnosis will not alter the treatment strategies and prognosis for these patients [11]. However, the utility of biopsy for DIPGs has been revaluated since the late 2000s, as those biopsy-based genomic studies of DIPGs has provided profound knowledge about this disease [12-14].

It is still unknown whether tumors arisen from different segments (such as the medulla oblongata, pons, and midbrain) of the brainstem but with same pathological diagnosis share the same rules of tumorigenesis and development. In 1990, Barkovich et al. [15] for the first time introduced the origin of tumor into the classification system and proposed novel classification guidelines based on different parameters, such as growth patterns (intrinsic/exophytic, diffuse/focal), the locations of tumor origin (midbrain, pons, medulla oblongata), and characteristics of the tumor itself (the degree of edema of the brainstem, tumor hemorrhage, or necrosis, with or without hydrocephalus). This classification only provides the classification foundation, but not reflects the biological characteristics of tumors from different segments of the brainstem, because brainstem glioma is a complex disease, and its imaging findings are various.

In the early 1990s, the role of surgery in midbrain glioma was well recognized [16-18]. Most midbrain gliomas grow focally, which can be divided into tectum, tegmental, and aqueduct gliomas, based on the original locations [19]. In tectum gliomas, the low- and high-grade tumors account for 85 and $15 \%$, respectively [20]. The lowgrade tectum gliomas grow extremely slow, and in the absence of surgery, patients are able to keep in longterm stable via V-P shunt or EVT operation [20, 21]. However, the high-grade tectum gliomas grow rapidly and have very short disease course. A more active approach is required for the treatment of this kind of tumor, and the surgery is one of the safe and feasible treatment strategies $[16,18,19]$. Although the tegmental glioma is usually exhibited in low-grade, its growth is significantly faster than that of tectum glioma, and its surgical risk is also greater than tectum gliomas. There are few reports about the aqueduct glioma, but the current available findings show that most aqueduct gliomas are low-grade tumors and surgical resection is safe and feasible for this tumor [19, 22].

Until the early 2000s, brainstem gliomas are gradually accepted as an highly heterogeneous entity. The surgical treatment system for brainstem glioma has been basically established under the guideline of radiological classification. Many treatment concepts and the results tend to be consensus, after the surgical resection or subtotal resection, the dorsal exophytic tumor patients can get long-term survival and even can be cured. Most cervicomedullary tumors are low-grade astrocytomas and can gain the good outcome after the operation. Most midbrain and medulla oblongata focal tumors are low-grade astrocytomas, and the prognosis of these tumors can be significantly improved by operation. However, focal tumors are rare in pons, and most of them are high-grade gliomas. The operation for this kind of tumor is safe, but is not able to improve the prognosis [23]. The patients with tectum gliomas normally need the treatment with V-P shunt or third ventriculostomy. The operation is not suitable for DIPG patients.

\section{Unsolved problems by conventional neuroimaging technology}

Until now, imaging technology has exhibited an outstanding role in precise positioning, demonstrating the growth pattern, determining the feasibility of operation, and navigating the precise position in the process of operation, during the practice of diagnosis and treatment in brainstem gliomas. However, we are still facing the problems in the aspects of qualitative diagnosis and the dynamic monitoring of treatment effect. For example, it is difficult to use the conventional MRI to distinguish WHO grade I pilocytic astrocytoma from WHO grade IV glioblastoma, gliomas from non-gliomas, neoplastic from non-neoplastic lesions [24-26]. Moreover, it is unable to detect earlier the response of tumor to radiotherapy and chemotherapy, for example difficulties for discriminating pseudo-progress, tumor recurrence, and radiation-induced necrosis [27]. In addition, it is unable to do the subdivision on DIPG using the imaging technology, for example, the radiological manifestations of child and adult DIPG patients are the same, but their prognosis and incidence are significantly different [28-30]. Moreover, the different ages of children also exhibit the significant differences, for example, the prognosis of pediatric patients with DIPG younger than 3-4 years old is better than elder ages [31-33]. 
Conventional imaging diagnostic sequence is facing the enormous confusion and challenges in determining the pathological types of glioma.

\section{The treatment of brainstem glioma promoted by molecular pathology}

The development of molecular pathology first answered the questions that are not able to be solved by neuroimaging at gene level. The tumor with similar imaging findings may have totally different molecular genetic events. For example, the molecular genetic event underlying pilocytic astrocytoma is the BRAF gene rearrangement [34]. However, the common genes that mutated in other brainstem gliomas are TP53, H3F3A, IDH1, PPM1D, and ACVR1 [35-40]. There are significant differences about the molecular genetics between children and adults DIPGs. The common mutated genes of children DIPGs are K27MH3F3A (70 \%) and ACVR1 (20-30 \%) [35, 37, 38]. The prognosis of DIPG patients with $K 27 M-H 3 F 3 A$ mutation is the worst [35]. ACVR1 mutation coexisted with $K 27 M$ $H 3.1$ mutation, but it excludes each other with $K 27 M-$ H3F3A mutation [38]. The DIPG patients with ACVR1 mutation are younger and have longer survival time [37, 38]. The common mutated genes for adult brainstem glioma are IDH1 and TP53. The mutations of IDH1 and K27M-H3F3A exclude each other, and they have opposite effects, and the brainstem glioma patients with IDH1 mutation have better prognosis [36]. Most brainstem gliomas do not have the MGMT promoter methylation, thus explaining why they are resistant to temozolomide and other alkylating agents [41, 42], and the mutations of PPM1D and TP53 would explain the reason for radiation resistance [36, 43]. The tumors with the same pathological types have distinct gene mutations between the brainstem and cerebral hemisphere. K27M-H3F3A mutation is mainly present in the brainstem and thalamus high-grade gliomas, while $G 34 V-H 3 F 3 A$ mutation is mainly present in the cerebral hemisphere gliomas [44]. PPM1D and ACVR1 mutations mainly occur in the brainstem gliomas, but are rare in supratentorial gliomas [36, 38]. The main mutation for brainstem pilocytic astrocytoma is the fusion mutation of BRAF-KIAA1549 [34], and that for sellar area pilocytic astrocytoma is the mutation of V600E BRAF [45]. All these molecular genetic findings confirm the essential difference between brainstem gliomas and supratentorial gliomas, which explain why chemotherapy is effective for supratentorial gliomas, but not for brainstem gliomas [42, 10].

In addition to elucidating the molecular mechanisms underlying the heterogeneity of brainstem gliomas, the development of the molecular pathology provides the potential therapeutic targets (such as ACVR1, PPM1D, $I D H 1, H 3 F 3 A$, and $B R A F$ ) for drug development in the future. We believe that with the advancement of molecular pathology on the brainstem gliomas, a comprehensive pathological classification will greatly improve the prognosis of brainstem gliomas.

\section{The advancement of neuroimaging and molecular imaging}

In the recent 10 years, the molecular pathology has gradually replaced the histopathology as the gold standard for guiding the personal diagnosis and treatment of gliomas. However, the diagnosis by molecular pathology only reflects the information of selected location, but not the whole tumor, and this method is limited for repeated sample collection, thus inducing the difficulties of molecular pathology in dynamic monitoring changes during the tumor treatment. In contrast, tumor imaging can reflect the whole information of tumor and also dynamically monitor the changes during the treatment process. It is necessary to combine these two disciplines if we want to take their advantages. Imaging genomics, imaging proteomics, and imaging metabolomics represent the new branch in science that link currently used imaging modalities to predict and correlate genomic, proteomic, and metabolic profiles in gliomas [46-49]. Among them, the fastest growing discipline is the imaging genomics in the filed of glioblastoma multiforme (GBM).

Raza et al. investigated the relationship between the degree of necrosis of GBM and gene expression profiles in 2004 and found that 26 genes are associated with GBM necrosis [46]. Diehn et al. [50] found that overexpression of EGFR is associated with the higher contrast-to-necrosis ratio. Pope et al. [51] confirmed that the expression of IL-8 and VEGF in completely enhancement GBM patients is significantly higher than that in incompletely enhancement patients. In 2011, Zinn et al. [52] identified the peritumoral MRI-FLAIR phenotype as an imaging surrogate for GBMs highly enriched in genes and miRNAs involved in cellular migration/invasion and specifically identified a gene and miRNA functional axis involved in invasion. These findings for the first time confirm that POSTN gene and its regulator miRNA-219 are associated with a highly significant decrease in survival, imaging characteristics of invasion, and a specific subclass of GBM, the mesenchymal subclass. Magnetic resonance perfusion (MRP) imaging is associated with the expression levels of pro-angiogenic genes [53]. However, these studies are still at statistical level, and their conclusions are not applied for the individual decision during the process of clinical practice. In addition, magnetic resonance spectroscopy (MRS) can detect carcinogenic product 2-hydroxyglutarate induced by the intratumoral $I D H 1 / 2$ gene mutation [54]. This is the technology that is currently able to assist personal therapy and observe the treatment efficacy. 
Because of its features of high sensitivity and targeting the metabolism, the positron emission tomography (PET)/CT has been utilized in the diagnosis and therapy guidance of brainstem gliomas and yielded its benefits comparing to the traditional imaging techniques. Pirotte et al. integrated PET/CT images into the planning for stereotactic biopsy procedures to direct the biopsy needle's trajectory to hypermetabolic foci of intrinsic infiltrative brainstem lesions in 20 children, and found that ${ }^{18}$ F-FDG and ${ }^{11} \mathrm{C}$-Methionine (MET) PET could help point out more accurate diagnostic yield for biopsy to get the tumor tissue for pathology determination than MRI alone. PET guidance could improve the sampling and reduce the numbers of sampling procedures. It was this study that strengthened the role of stereotactically guided biopsy procedures which was once questioned in intrinsic infiltrative brainstem lesions [55]. The tumor metabolism molecular imaging could yield some prognostic marker for brainstem gliomas. A report of Pediatric Brain Tumor Consortium from a research of 40 children of newly diagnosed diffuse intrinsic brainstem gliomas found that the patients with ${ }^{18}$ F-FDG uptake involves at least half the tumor had the poorer survival than those with uptake in less than $50 \%$ of the tumor [56]. In the future, PET/ MRI, which combines the advantage of high sensitivity of PET and high resolution of MRI, will be used more widely than before [47]. What is more, with the identification of tumor-specific proteins and genes, more and more imaging tracers, including antibody, peptides, and small molecular chemicals, will be designed for a great breakthrough in cancer diagnosis, differential diagnosis, and dynamic monitoring.

A great progress in the genomics of brainstem glioma has been made in the recent 5 years, such as the $B R A F, H 3 F 3 A$, $A C V R 1, P P M 1 D$, and IDH1 genes have been successively identified [34-40]. The imaging genomics of brainstem glioma is still completely unknown. We believe that rapid development in this field will be gained in the future.

\section{Conclusion}

Brainstem glioma is a group of highly heterogeneous disease, and the cooperation among multiple disciplines is necessary to cure this disease. Improving the molecular pathological classification of brainstem glioma is the basis of the personal diagnosis and therapy. It is necessary to combine the imaging with molecular pathology, thus developing novel method that is noninvasive, comprehensive, and able to dynamically monitor the treatment of cancer.

\section{Competing interests}

The authors declare that they have no competing interests.

\section{Authors' contributions}

All authors did the conception and design of the study, critically revised the article, and reviewed the submitted version of the manuscript. LZ drafted the article and approved the final version of the manuscript on behalf of all authors. All authors read and approved the final manuscript.

\section{Acknowledgements}

The authors would like to thank Dr. Cheng Xu for his help in proofreading this manuscript. The study was supported by the National Key Technology Research and Development Program of the Ministry of Science and Technology of China (grant No. 2013BAI09B03).

\section{Author details}

${ }^{1}$ Department of Neurosurgery, Beijing Tiantan Hospital, Capital Medical University, Tiantanxili 6, Dongcheng District, Beijing 100050, China. ${ }^{2}$ China National Clinical Research Center for Neurological Disease, Tiantanxili 6, Dongcheng District, Beijing 100050, China. ${ }^{3}$ Medical Center, Tsinghua University, Haidian District, Beijing 100084, China.

Received: 26 May 2015 Accepted: 6 July 2015

Published online: 18 September 2015

\section{References}

1. Ingraham FD, Maston DD. Neurosurgery of infancy and childhood. 1969.

2. Hoffman HJ, Becker L, Craven M. A clinically and pathologically distinct group of benign brain stem gliomas. Neurosurgery. 1980;7(3):243-8.

3. Fisher PG, Breiter SN, Carson BS, Wharam MD, Williams JA, Weingart JD, et al. A clinicopathologic reappraisal of brain stem tumor classification. Cancer. 2000;89(7):1569-76.

4. Stroink AR, Hoffman HJ, Hendrick EB, Humphreys RP. Diagnosis and management of pediatric brain-stem gliomas. J Neurosurg. 1986;65(6):745-50.

5. Epstein F. A staging system for brain stem gliomas. Cancer. 1985;56(S7):1804-6.

6. Epstein F, McCleary EL. Intrinsic brain-stem tumors of childhood: surgical indications. J Neurosurg. 1986;64(1):11-5.

7. Epstein F, Wisoff J. Intra-axial tumors of the cervicomedullary junction. J Neurosurg. 1987;67(4):483-7.

8. Epstein FJ, Farmer J-P. Brain-stem glioma growth patterns. J Neurosurg. 1993;78(3):408-12.

9. Scherer H. Structural development in gliomas. Am J Cancer. 1938;34(3):333-51.

10. Hargrove $D$, Bartels $U$, Bouffet E. Diffuse brainstem glioma in children: critical review of clinical trials. Lancet Oncol. 2006;7(3):241-8. doi:10.1016/s1470-2045(06)70615-5.

11. Albright AL, Packer RJ, Zimmerman R, Rorke LB, Boyett J, Hammond GD. Magnetic resonance scans should replace biopsies for the diagnosis of diffuse brain stem gliomas: a report from the Children's Cancer Group. Neurosurgery. 1993;33(6):1026-30.

12. Hargrave D. Diffuse brainstem gliomas in children: should we or shouldn't we biopsy? Br J Neurosurg. 2008;22(5):624. doi:10.1080/02688690802484405.

13. Wilkinson R, Harris J. Moral and legal reasons for altruism in the case of brainstem biopsy in diffuse glioma. Br J Neurosurg. 2008;22(5):617-8. doi:10.1080/02688690802482896.

14. Bartels U, Hawkins C, Vezina G, Kun L, Souweidane M, Bouffet E. Proceedings of the diffuse intrinsic pontine glioma (DIPG) Toronto Think Tank: advancing basic and translational research and cooperation in DIPG. J Neurooncol. 2011;105(1):119-25. doi:10.1007/s11060-011-0704-4.

15. Barkovich A, Krischer J, Kun L, Packer R, Zimmerman R, Freeman C, et al. Brain stem gliomas: a classification system based on magnetic resonance imaging. Pediatr Neurosurg. 1990;16(2):73-83.

16. Pendl G, Vorkapie P, Koniyama M. Microsurgery of midbrain lesions. Neurosurgery. 1990;26(4):641-8.

17. Vandertop WP, Hoffman HJ, Drake JM, Humphreys RP, Rutka JT, Amstrong DC, et al. Focal midbrain tumors in children. Neurosurgery. 1992;31(2):186-94.

18. Bognar L, Fischer C, Turjman F, Michel F, Villanyi E, Mottolese C, et al. Tectal plate gliomas Part III: apparent lack of auditory consequences of unilateral inferior collicular lesion due to localized glioma surgery. Acta Neurochir. 1994;127(3-4):161-5.

19. Wang CC, Zhang JT, Liu A, Sun B, Zhao YL. Surgical treatment of primary midbrain gliomas. Surg Neurol. 2000;53(1):41-51. doi:10.1016/s00903019(99)00165-2. 
20. Daglioglu E, Cataltepe O, Akalan N. Tectal gliomas in children: the implications for natural history and management strategy. Pediatr Neurosurg. 2003;38(5):223-31. doi:69823.

21. Yeh DD, Warnick RE, Ernst RJ. Management strategy for adult patients with dorsal midbrain gliomas. Neurosurgery. 2002;50(4):735-8. discussion 8-40.

22. Pool JL. Gliomas in the region of the brain stem*. J Neurosurg. 1968;29(2):164-7.

23. Bricolo A. Surgical management of intrinsic brain stem gliomas. Oper Tech Neurosurg. 2000;3(2):137-54. doi:10.1053/oy.2000.6559.

24. Kumar AJ, Leeds NE, Kumar VA, Fuller GN, Lang FF, Milas Z, et al. Magnetic resonance imaging features of pilocytic astrocytoma of the brain mimicking high-grade gliomas. J Comput Assist Tomogr. 2010;34(4):601-11. doi:10.1097/RCT.0b013e3181d77d52.

25. Scott RM. Surgical outcome following resection of contrast-enhanced pediatric brainstem gliomas. Pediatr Neurosurg. 2004;40(2):99.

26. Zhang S, Wang X, Liu X, Ju Y, Hui X. Brainstem gangliogliomas: a retrospective series. J Neurosurg. 2013;118(4):884-8. doi:10.3171/2013.1.JNS121323.

27. Alexiou GA, Tsiouris S, Kyritsis AP, Voulgaris S, Argyropoulou MI, Fotopoulos AD. Glioma recurrence versus radiation necrosis: accuracy of current imaging modalities. J Neurooncol. 2009;95(1):1-11. doi:10.1007/s11060-009-9897-1.

28. Selvapandian S, Rajshekhar V, Chandy MJ. Brainstem glioma: comparative study of clinico-radiological presentation, pathology and outcome in children and adults. Acta Neurochir (Wien). 1999;141(7):721-6.

29. Paugh BS, Qu C, Jones C, Liu Z, Adamowicz-Brice M, Zhang J, et al. Integrated molecular genetic profiling of pediatric high-grade gliomas reveals key differences with the adult disease. J Clin Oncol. 2010;28(18):3061-8. doi:10.1200/JCO.2009.26.7252.

30. Gilheeney SW, Kieran MW. Differences in molecular genetics between pediatric and adult malignant astrocytomas: age matters. Future Oncol. 2012;8(5):549-58.

31. Broniscer A, Laningham FH, Sanders RP, Kun LE, Ellison DW, Gajjar A. Young age may predict a better outcome for children with diffuse pontine glioma. Cancer. 2008;113(3):566-72.

32. Wagner S, Warmuth-Metz M, Emser A, Gnekow AK, Strater R, Rutkowski S, et al. Treatment options in childhood pontine gliomas. J Neurooncol. 2006;79(3):281-7. doi:10.1007/s11060-006-9133-1.

33. Jackson S, Patay Z, Howarth R, Pai Panandiker AS, Onar-Thomas A, Gajjar A, et al. Clinico-radiologic characteristics of long-term survivors of diffuse intrinsic pontine glioma. J Neurooncol. 2013;114(3):339-44. doi:10.1007/s11060-013-1189-0.

34. Horbinski C, Hamilton RL, Nikiforov Y, Pollack IF. Association of molecular alterations, including BRAF, with biology and outcome in pilocytic astrocytomas. Acta Neuropathol. 2010;119(5):641-9. doi:10.1007/s00401-009-0634-9.

35. Khuong-Quang DA, Buczkowicz P, Rakopoulos P, Liu XY, Fontebasso AM, Bouffet $E$, et al. K27M mutation in histone H3.3 defines clinically and biologically distinct subgroups of pediatric diffuse intrinsic pontine gliomas. Acta Neuropathol. 2012;124(3):439-47. doi:10.1007/s00401-012-0998-0.

36. Zhang L, Chen LH, Wan H, Yang R, Wang Z, Feng J, et al. Exome sequencing identifies somatic gain-of-function PPM1D mutations in brainstem gliomas. Nat Genet. 2014;46(7):726-30. doi:10.1038/ng.2995.

37. Wu G, Diaz AK, Paugh BS, Rankin SL, Ju B, Li Y, et al. The genomic landscape of diffuse intrinsic pontine glioma and pediatric non-brainstem high-grade glioma. Nat Genet. 2014;46(5):444-50. doi:10.1038/ng.2938.

38. Taylor KR, Mackay A, Truffaux N, Butterfield YS, Morozova O, Philippe C, et al. Recurrent activating ACVR1 mutations in diffuse intrinsic pontine glioma. Nat Genet. 2014;46(5):457-61. doi:10.1038/ng.2925.

39. Fontebasso AM, Papillon-Cavanagh S, Schwartzentruber J, Nikbakht H, Gerges N, Fiset PO, et al. Recurrent somatic mutations in ACVR1 in pediatric midline high-grade astrocytoma. Nat Genet. 2014;46(5):462-6. doi:10.1038/ng.2950.

40. Buczkowicz P, Hoeman C, Rakopoulos P, Pajovic S, Letourneau L, Dzamba M, et al. Genomic analysis of diffuse intrinsic pontine gliomas identifies three molecular subgroups and recurrent activating ACVR1 mutations. Nat Genet. 2014:46(5):451-6. doi:10.1038/ng.2936.

41. Oka H, Utsuki S, Tanizaki Y, Hagiwara H, Miyajima Y, Sato K, et al. Clinicopathological features of human brainstem gliomas. Brain Tumor Pathol. 2013;30(1):1-7. doi:10.1007/s10014-012-0099-8.
42. Frazier JL, Lee J, Thomale UW, Noggle JC, Cohen KJ, Jallo Gl. Treatment of diffuse intrinsic brainstem gliomas: failed approaches and future strategies: A review. J Neurosurg Pediatr. 2009;3(4):259-69.

43. Mandell LR, Kadota R, Freeman C, Douglass EC, Fontanesi J, Cohen ME, et al. There is no role for hyperfractionated radiotherapy in the management of children with newly diagnosed diffuse intrinsic brainstem tumors: results of a Pediatric Oncology Group phase III trial comparing conventional vs. hyperfractionated radiotherapy. Int J Radiat Oncol Biol Phys. 1999;43(5):959-64.

44. Sturm D, Witt H, Hovestadt V, Khuong-Quang DA, Jones DT, Konermann C, et al. Hotspot mutations in $\mathrm{H} 3 \mathrm{~F} 3 \mathrm{~A}$ and IDH1 define distinct epigenetic and biological subgroups of glioblastoma. Cancer Cell. 2012;22(4):425-37. doi:10.1016/j.ccr.2012.08.024.

45. Jones DT, Gronych J, Lichter P, Witt O, Pfister SM. MAPK pathway activation in pilocytic astrocytoma. Cell Mol Life Sci. 2012;69(11):1799-811.

46. Zinn PO, Colen RR. Imaging genomic mapping in glioblastoma. Neurosurgery. 2013;60:126-30.

47. Puttick S, Bell C, Dowson N, Rose S, Fay M. PET, MRI, and simultaneous PET/MRI in the development of diagnostic and therapeutic strategies for glioma. Drug Discov Today. 2015;20(3):306-17. doi:10.1016/j.drudis.2014.10.016.

48. Genomics of brain tumor imaging. doi:10.1016/j.nic.2014.09.006.

49. Imaging genomics of glioblastoma state of the art bridge between genomics and neuroradiology. doi:10.1016/j.nic.2014.09.010.

50. Diehn M, Nardini C, Wang DS, McGovern S, Jayaraman M, Liang Y, et al. Identification of noninvasive imaging surrogates for brain tumor gene-expression modules. Proc Natl Acad Sci U S A. 2008;105(13):5213-8. doi:10.1073/pnas.0801279105.

51. Pope WB, Chen JH, Dong J, Carlson MR, Perlina A, Cloughesy TF, et al. Relationship between gene expression and enhancement in glioblastoma multiforme: exploratory dna microarray analysis 1. Radiology. 2008;249(1):268-77.

52. Zinn PO, Mahajan B, Sathyan P, Singh SK, Majumder S, Jolesz FA, et al. Radiogenomic mapping of edema/cellular invasion MRI-phenotypes in glioblastoma multiforme. PLoS One. 2011;6(10):e25451. doi:10.1371/journal.pone.0025451.

53. Jain R, Poisson L, Narang J, Scarpace L, Rosenblum ML, Rempel S, et al. Correlation of perfusion parameters with genes related to angiogenesis regulation in glioblastoma: a feasibility study. AJNR Am J Neuroradiol. 2012;33(7):1343-8. doi:10.3174/ajnr.A2956.

54. Choi C, Ganji SK, DeBerardinis RJ, Hatanpaa KJ, Rakheja D, Kovacs Z, et al. 2-hydroxyglutarate detection by magnetic resonance spectroscopy in IDH-mutated patients with gliomas. Nat Med. 2012;18(4):624-9. doi:10.1038/nm.2682.

55. Pirotte BJ, Lubansu A, Massager N, Wikler D, Goldman S, Levivier M. Results of positron emission tomography guidance and reassessment of the utility of and indications for stereotactic biopsy in children with infiltrative brainstem tumors. J Neurosurg. 2007;107(5 Suppl):392-9. doi:10.3171/PED-07/11/392.

56. Zukotynski KA, Fahey FH, Kocak M, Alavi A, Wong TZ, Treves ST, et al. Evaluation of 18F-FDG PET and MRI associations in pediatric diffuse intrinsic brain stem glioma: a report from the Pediatric Brain Tumor Consortium. J Nucl Med. 2011;52(2):188-95. doi:10.2967/jnumed.110.081463.

\section{Submit your next manuscript to BioMed Central and take full advantage of:}

- Convenient online submission

- Thorough peer review

- No space constraints or color figure charges

- Immediate publication on acceptance

- Inclusion in PubMed, CAS, Scopus and Google Scholar

- Research which is freely available for redistribution 